\title{
Sistema fotodetector econômico para utilização em laboratórios de ensino e pesquisa
}

\author{
A low-cost photodetector system for research and educational laboratory
}

\author{
Henrique G. Gutierre ${ }^{1}$, Marlon S. Ribeiro ${ }^{1}$, Luiz A. A. Pereira ${ }^{1}$, Gerson K. da Cruz $^{1}$, Rozane de F. \\ Turchiello $^{2}$, Sergio L. Gómez ${ }^{* 1}$ \\ ${ }^{1}$ Departamento de Física, Universidade Estadual de Ponta Grossa, Ponta Grossa, PR, Brasil \\ ${ }^{2}$ Departamento de Física, Universidade Tecnológica Federal do Paraná, Ponta Grossa, PR, Brasil
}

Recebido em 24 de Novembro, 2016. Revisado em 16 de Dezembro, 2016. Aceito em 20 de Dezembro, 2016.

\begin{abstract}
Um sistema fotodetector para a sua utilização em medidas quantitativas da irradiância ou potência de um feixe de luz laser precisa ter uma resposta linear. Dispositivos com essa finalidade podem ser adquiridos comercialmente, mas possuem custo elevado. Nesta comunicação é proposta a construção de um sistema fotodetector a partir de um resistor dependente de luz (LDR), o qual é um elemento cuja resposta é não-linear. Nesse caso, para sua utilização necessitamos de uma calibração prévia do sistema. O desempenho do novo sistema em experiências de quantificação da potência da luz em condições estacionárias foi avaliada comparando o resultado da determinação da largura de um feixe de laser com perfil espacial gaussiano mediante a técnica da lâmina, com o obtido por um fotodetector comercial.
\end{abstract}

Palavras-chave: óptica, LDR, fotodetector.

A photodetector for using in quantitative measurements of the irradiance or power of a laser beam needs to have a linear response. Devices with this property can be acquired but usually they are expensive. In this paper we propose the construction of a photodetector that employs a Light Dependent Resistor (LDR), which has a nonlinear response. In this situation, for using such a photodetector it is necessary a previous calibration. The performance of the new system in experiments that need to quantify the radiant power in stationary conditions was evaluated by comparing the results of the measurement of the radius of a gaussian beam through the knife-edge technique, with the obtained with a commercial linear photodetector.

Keywords: optics, LDR, photodetector.

\section{Introdução}

Nos laboratórios de ensino de física, as experiências de óptica formam parte do núcleo fundamental de atividades básicas em nível introdutório nos cursos que possuem a disciplina Física nos seus currículos. Em geral o custo dos equipamentos necessários nas experiências de óptica diminuiu notavelmente mas, ainda assim, alguns itens permanecem com custo relativamente elevado. Nesta lista estão os fotodetectores utilizados para a quantificação da intensidade luminosa em experimentos que exigem tal determinação. Uma propriedade fundamental que um

*Endereço de correspondência: sgomez@uepg.br sistema fotodetector, com a finalidade mencionada anteriormente, deve ter é o de apresentar uma resposta linear em função da energia radiante incidente nele. Os fotodetectores lineares mais amplamente utilizados possuem um elemento semicondutor de silício ( $\mathrm{Si}$ ). Visando contribuir não só para tornar os laboratórios de óptica mais acessíveis do ponto de vista econômico, mas também propiciar uma formação mais completa dos alunos participando do processo de construção e aferimento de um equipamento de grande utilidade num laboratório de ensino e de pesquisa, propomos a elaboração de um fotodetector baseado na utilização de um LDR (Light Dependent Resistor), um elemento cuja resistência 
elétrica depende da energia radiante incidente sobre ele.

\section{LDR}

O LDR ou célula fotocondutiva é um dispositivo fotossensível normalmente fabricado a partir de semicondutores como sulfato de cádmio (CdS) ou seleneto de cádmio (CdSe), entre outros. São fabricados através da deposição de uma linha de CdS ou CdSe em vai-e-vem ("zig-zag") sobre um substrato isolante cerâmico entre dois elementos condutores (Fig. 1) e cujo objetivo é o de atingir valores específicos de resistência e potência nominais para o dispositivo. Para entendermos seu funcionamento devemos lembrar que um semicondutor possui um pequeno número de portadores de carga na banda de condução quando na temperatura ambiente. Entretanto, quando foto-estimulados com luz de energia superior à energia de gap do semicondutor, há a criação de novos portadores de carga a partir da transição de elétrons da banda de valência para a banda de condução. Como consequência desse processo ocorre um aumento da condutividade elétrica do dispositivo, ou em outras palavras, uma queda de sua resistência elétrica. O LDR é, portanto, um dispositivo que possui uma resistência elétrica dependente da irradiância $I_{L D R}$ do feixe, onde a irradiância $I$ é definida como a energia média por unidade de área e unidade de tempo, que incide no LDR. Possui resposta não-linear e sensibilidade lenta devido a processos de recombinação que ocorrem quando a luz é removida. Dentre as características a serem ressaltadas estão ainda a resistência do LDR quando nenhuma luz incide sobre ele (resistência no escuro) e a resistência de saturação quando submetido a condição de iluminação intensa. Finalmente, é importante destacar que o comprimento de onda da luz para o qual o LDR apresenta maior sensibilidade dependerá do material do qual ele é construído (Fig. 2).

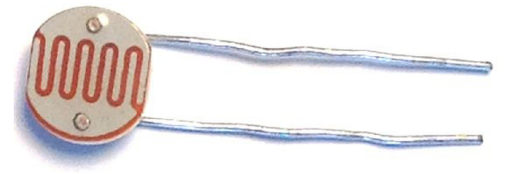

Figura 1: Imagem de um LDR.

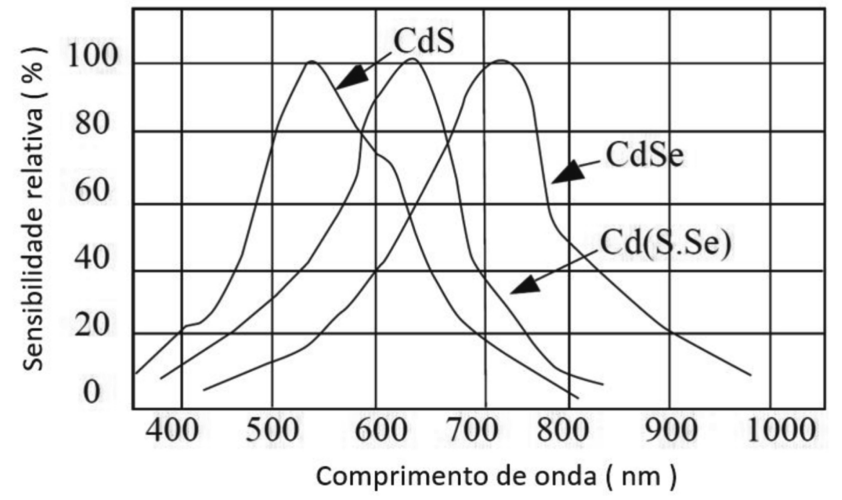

Figura 2: Gráfico da sensibilidade em relação ao comprimento de onda da luz de vários tipos de LDR contendo cádmio. Fonte: https://www.token.com.tw

\section{Materiais e método}

O sistema fotodetector consiste de um circuito no qual um resistor é ligado em série com o LDR. Como a resistência elétrica do LDR diminui com a irradiância do feixe de luz que incide nele, aumenta a diferença de potencial elétrico no resistor $\left(V_{R}\right)$. Assim $V_{R}$ é uma função crescente da irradiância mas não necessariamente linear. Um esquema do sistema fotodetector que utiliza o LDR é mostrado na Fig. 3.

Para que o sistema fotodetector baseado em um LDR possa ser usado para quantificar a energia radiante de um feixe ele deve ser calibrado. Para tal fim pode ser usado um laser de potência $P_{o}$ conhecida e um filtro polarizador sobre um suporte giratório. Para uma energia radiante distribuída sobre uma área transversal definida, pode-se escrever $I_{o} \propto P_{o}$. Se o laser for linearmente polarizado, será necessário somente um filtro polarizador. Se o laser não for linearmente polarizado, serão necessários dois filtros

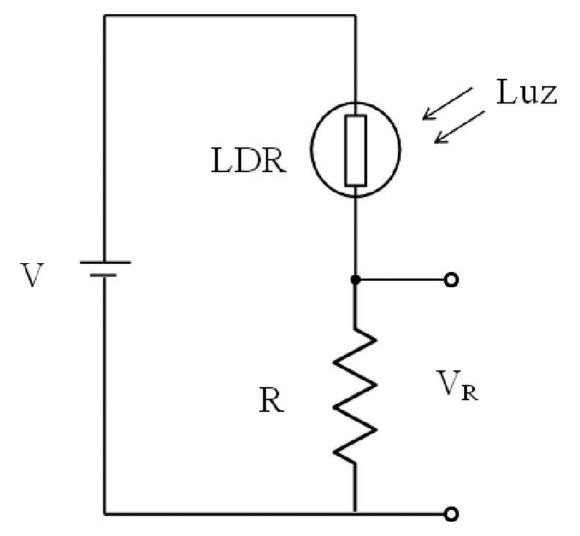

Figura 3: Esquema do sistema fotodetector. 
polarizadores, um fixo para polarizar o feixe de luz emitido pelo laser e um segundo filtro sobre o suporte giratório para controlar a intensidade do feixe. Se um feixe de luz linearmente polarizada proveniente de um laser de potência $P_{o}$ e irradiância $I_{o}$ incide num polarizador linear posicionado sobre o suporte giratório, a irradiância $I$ da luz transmitida por este polarizador é dada pela regra do cosseno ao quadrado, também conhecida como lei de Malus e mostrada na equação (1) [1,2]:

$$
I=I_{o} \cos ^{2} \theta
$$

onde $\theta$ é o ângulo entre a direção de polarização do feixe e a direção de polarização do polarizador sobre o suporte giratório. Supondo que o polarizador não altere a área do feixe, podemos escrever $I \propto P$, onde $P$ é a potência associada ao feixe após o polarizador. Se focalizamos o feixe de luz sobre o LDR, a área sobre a qual a energia radiante é depositada diminue mas a irradiância sobre o LDR, $I_{L D R}$, continuará sendo proporcional a $P$. Assim a tensão $V_{R}$ será uma função da potência $P$ da luz que atinge o LDR. A tensão $V_{R}$ pode ser lida diretamente por meio de um multímetro ou através de um sistema de aquisição de dados que use um conversor de sinal analógico/digital (A/D) o qual transforma um sinal analógico, como uma voltagem elétrica, num sinal digital. No sistema proposto, o sinal $V_{R}$ é lido utilizando a plataforma Arduino [3, modelo Leonardo, o qual possui um conversor A/D de 10 bits. Dessa forma, em resposta ao valor analógico de entrada $V_{R}$, o conversor retorna um valor numérico $N$ entre 0 e 1023, o qual é diretamente proporcional ao valor analógico de entrada. Portanto, para a utilização do sistema basta que façamos uma calibração do fotodetector através da construção de um gráfico $P$ vs $V_{R}$ ou $P$ vs $N$. Utilizando-se um programa de tratamento de dados, como Origin ${ }^{\circledR}$, efetua-se um ajuste dos dados considerando que a relação entre ambas grandezas, $P$ vs $V_{R}$ ou $P$ vs $N$, segue, por exemplo, uma lei de potência. Assim, quando utilizado o sistema fotodetector numa medida, a leitura de $V_{R}$ ou $N$ deve ser convertida em potência $P$ por meio da curva de calibração. A resistência elétrica do LDR tem dependência não-linear com a intensidade da luz incidente nele [4, sendo o seu comportamento, em geral, diferente em distintas faixas de valores da intensidade [5]. Assim, a escolha da função de ajuste da curva de calibração dependerá do tipo de LDR e da faixa de potência utilizada. Na Fig. 4 é

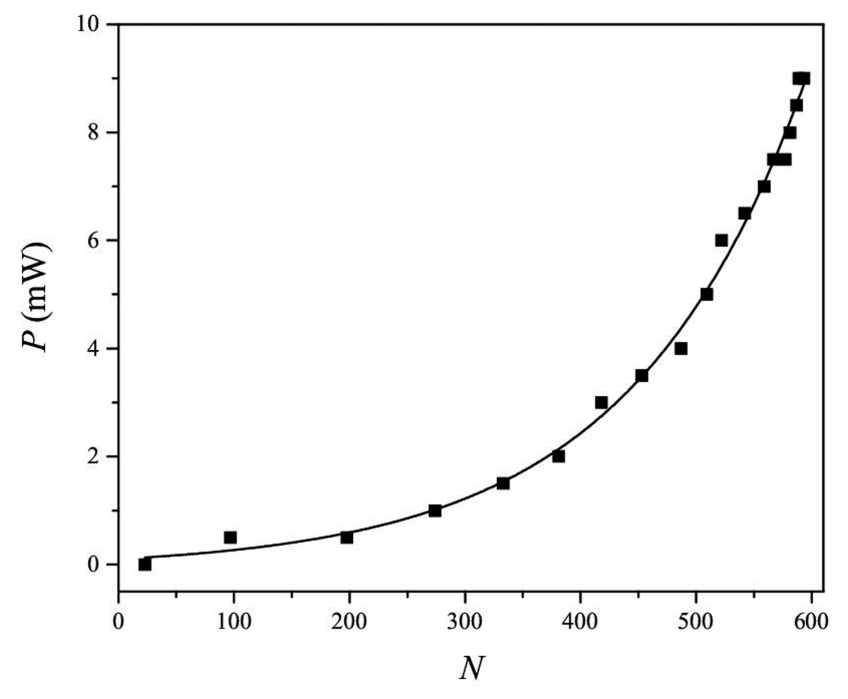

Figura 4: Curva de calibração $P$ vs $N$ do sistema fotodetector. A linha contínua representa um ajuste dos dados experimentais à função dada na Eq. 2.

mostrada a dependência do sinal do conversor A/D com a potência incidente do feixe de luz sobre o LDR utilizado nesse trabalho, na qual foi usada a seguinte função de ajuste:

$$
P=a+b e^{\frac{N}{c}}
$$

onde $a, b$ e $c$ são parâmetros do ajuste e $e$ é a base do logaritmo natural ou neperiano. A escolha da função mostrada na equação (2) foi baseada no teste do $\chi_{\text {red }}^{2}$, o qual permite a determinação da função, entre várias funções possíveis, que melhor ajusta um determinado conjunto de dados [6]. Um cuidado especial que deve se ter na utilização do sistema com o LDR é em relação à direção de incidência do feixe com a normal à superfície. A transmissividade da energia radiante numa interface dada entre dois meios, definida como a razão entre a energia transmitida e a incidente, depende do ângulo entre a direção normal à superfície do meio e a direção de incidência do feixe [7]. Portanto, cuidado especial deve ser tomado para que não se altere a posição nem a orientação do LDR durante a realização de uma mensuração.

O sistema fotodetector foi posto a prova determinando a largura de um feixe gaussiano 8. Os lasers comerciais usualmente emitem um feixe de luz com irradiância não uniforme, cuja distribuição espacial corresponde ao modo fundamental do oscilador óptico, conhecido como $\mathrm{TEM}_{00}$ ou gaussiano. A dependência espacial do feixe gaussiano é dada 
pela equação (3):

$$
I=I_{m} e^{-\frac{r^{2}}{w^{2}}}
$$

onde $r$ é a distância radial em relação ao eixo de propagação do feixe, $I_{m}$ é a irradiância do feixe sobre o eixo de propagação $(r=0)$ e $w$ é a largura do feixe. A divergência do feixe leva a uma mudança na largura do mesmo ao longo do eixo de propagação. Nesse trabalho, a determinação da largura do feixe foi feita num ponto indeterminado ao longo do eixo de propagação.

A medida da largura do feixe pode ser feita por meio do método da lâmina [9, 10, o qual consiste na quantificação da luz que atinge o detector como função da posição de uma lâmina que é deslocada perpendicularmente à direção de propagação do feixe, por meio de um parafuso micrométrico. Para uma medida precisa, após a lâmina é posicionada uma lente a qual focaliza o feixe sobre o fotodetector. O sinal do fotodetector quando a borda da lâmina está na posição $x, V_{R}(x)$ ou $N(x)$, será proporcional à potência radiante total que não é bloqueada pela lâmina, ou seja:

$$
\begin{aligned}
& V_{R}(x), N(x) \stackrel{\text { Eq. } 2}{\longrightarrow} P(x) \\
& =\int_{x}^{+\infty} \int_{-\infty}^{+\infty} I_{m} e^{-\frac{x^{\prime 2}+y^{2}}{w^{2}}} d x^{\prime} d y
\end{aligned}
$$

onde a posição $x=0$ é o ponto de partida da varredura. A integral dupla da equação (4) é proporcional a $\operatorname{erfc}(x / w)$, onde a função $\operatorname{erfc}(x)$, mostrada na equação (5), é denominada função erro complementar [11:

$$
\operatorname{erfc}(x) \equiv \frac{2}{\pi} \int_{x}^{+\infty} e^{-t^{2}} d t
$$

Pelo teorema fundamental do cálculo, a derivada da função $\operatorname{erfc}(x / w)$ é proporcional à função gaussiana $\exp \left(-r^{2} / w^{2}\right)$.

\section{Resultados Experimentais}

A Fig. 5 mostra o resultado da determinação do perfil espacial do feixe de um laser de HeNe (Melles Griot, CW, $16 \mathrm{~mW}$, linearmente polarizado) por meio do método da lâmina utilizando o fotodetector Det550 (Optron) o qual utiliza um fotodiodo de Si. A Fig. 5a mostra o sinal $V$ do fotodetector, uma diferença de potencial elétrico proporcional à a)

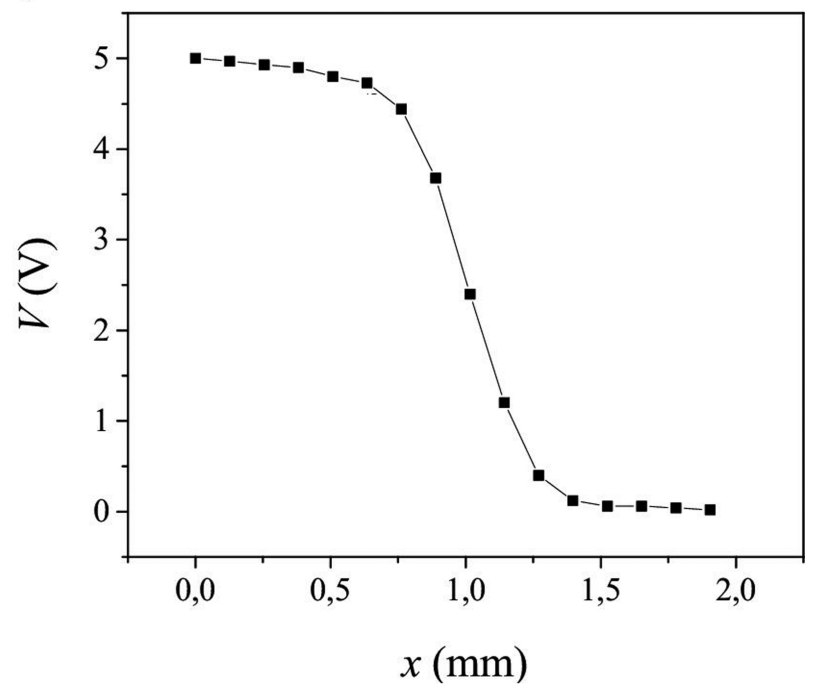

b)

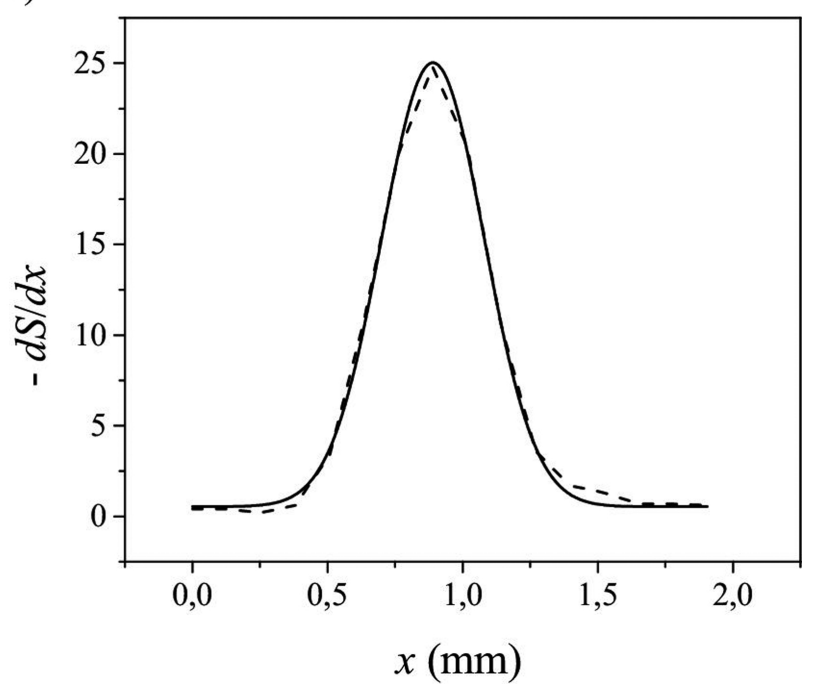

Figura 5: Determinação do perfil de um laser de $\mathrm{HeNe}$ obtido pelo método da lâmina usando um fotodetector que utiliza um fotodiodo de Si. a) Gráfico da transmitância da lâmina. A linha é um guia para os olhos; b) em linha tracejada é mostrada a derivada do sinal do fotodetector e em linha contínua o ajuste por uma função gaussiana.

irradiância da parte do feixe que não foi bloqueada pela lâmina, em função da posição da lâmina. Os dados são obtidos partindo de uma posição onde o feixe está completamente desobstruido, bloqueandoo de forma crescente. A curva assim obtida é proporcional à função erro complementar. A Fig. 5b mostra a negativa da derivada do sinal do fotodetector e um ajuste desta por uma função gaussiana. Do ajuste da oposta à derivada de $V(x)$ por uma função gaussiana, o valor obtido da largura do feixe é $w=0,378 \pm 0,005 \mathrm{~mm}$. 
A Fig. 6 por sua vez mostra o resultado da determinação da largura do feixe do mesmo laser de HeNe mas utilizando o sistema fotodetector com o LDR. A Fig. 6a mostra o sinal do sistema fotodetector, retificado pela função de calibração, em função da posição da lâmina. Por sua vez, a Fig. 6b mostra a derivada do sinal do sistema fotodetector e um ajuste desta por uma função gaussiana. Do ajuste do oposto da derivada de $N(x)$ por uma função gaussiana, o valor obtido da largura do feixe é $w=0,372 \pm 0,003 \mathrm{~mm}$.

a)

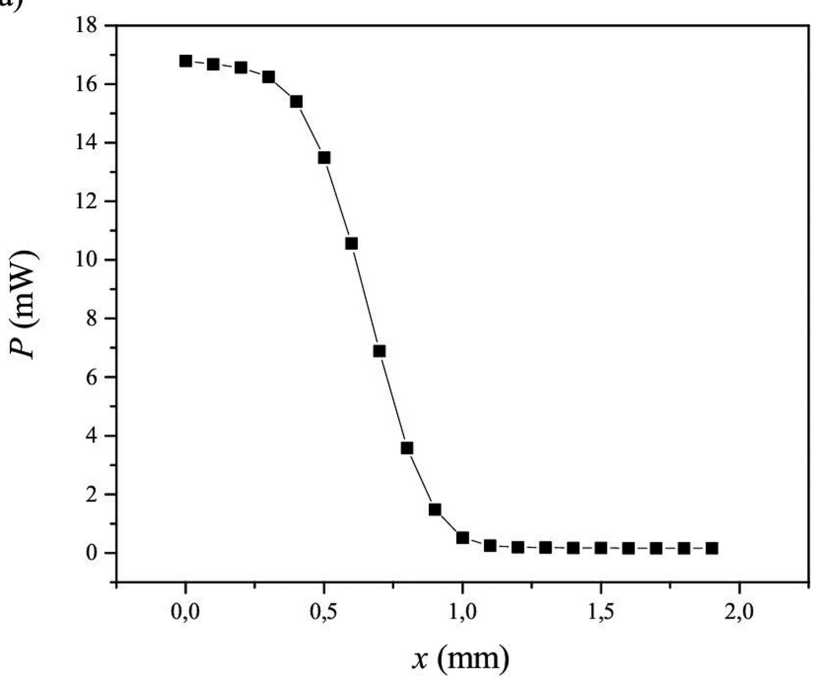

b)

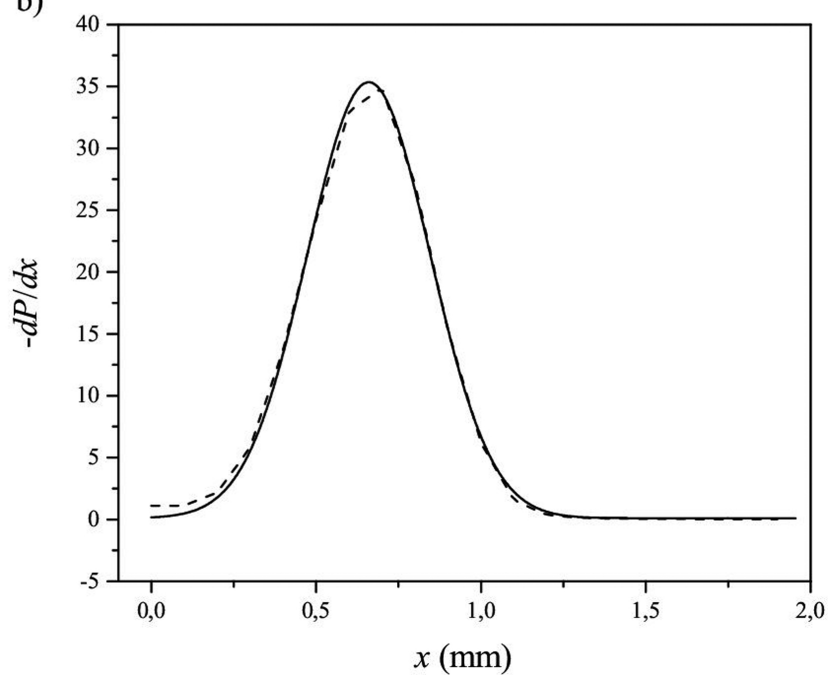

Figura 6: Determinação do perfil de um laser de $\mathrm{HeNe}$ obtido pelo método da lâmina utilizando um LDR como elemento fotossensível. a) Gráfico da transmitância da lâmina. A linha é um guia para os olhos; b) em linha tracejada é mostrada a derivada do sinal do sistema fotodetector e em linha contínua o ajuste por uma função gaussiana.
Os resultados mostram que o fotodetector de $\mathrm{Si}$ e o sistema fotodetector baseado em LDR fornecem valores aproximadamente iguais para a largura de um feixe gaussiano, o qual demonstra a viabilidade do sistema fotodetector construído utilizando um LDR como elemento fotossensível. Assim, um sistema baseado em LDR consitui um instrumento de medida preciso em situações onde é necessário quantificar a energia radiante em condições estacionárias.

\section{Conclusões}

Nesta comunicação apresentamos um sistema fotodetector de baixo custo construído a partir de um LDR. Demonstramos sua confiabilidade através da determinação do perfil do feixe de um laser de feixe gaussiano. Esse resultado mostrou que o sistema pode ser utilizado tanto em laboratário de ensino quanto de pesquisa quando a determinação da irradiância de um feixe de luz não exigir a resolução temporal. Assim esperamos contribuir tanto para o fortalecimento do ensino de física, em particular da área de óptica, quanto para as pesquisas que envolvam sistemas ópticos.

\section{Agradecimentos}

Os autores agradecem às agências de fomento CNPq, CAPES e Fundação Araucária, à Universidade Estadual de Ponta Grossa e ao Instituto Nacional de Ciência e Tecnologia em Fluidos Complexos (INCT$\mathrm{FCx}$ ) pelo apoio financeiro.

\section{Referências}

[1] R. Resnick, D. Halliday e J. Walker, Fundamentos de Física, $8^{\mathrm{a}}$ ed, (LTC, Rio de Janeiro, 2008), vol. 4.

[2] E. Hecht, Optics (Addison Wesley, San Francisco, 2002), 4th ed, cap. 8, sec. 2.

[3] Para uma visão geral do que é Arduino, os diferentes modelos de plataforma, instalação do software, manuais e demais informações, pode-se visitar o site do projeto original: www.arduino.cc Para aplicações em Física pode-se consultar: R.F. de Rodrigues e S.L. Souza Cunha, Textos de Apoio ao Professor de Física 25, (4) (2014), disponível em www.if.ufrgs.br/public/tapf/

[4] F. Ciampini, P.S. Scarazzato, A.A.R. Neves, D.C.L. Pereira and M.H. Yamanaka, Sol. Energy 81, 1187 (2007).

[5] D.F. da Silva and D. Acosta-Avalos, Sensors 6, 514 (2006). 
[6] J.H. Vuolo, Fundamentos da Teoria de Erros (Edgard Blücher, São Paulo, 1996), 2ª ed., cap. 14, sec. 3 e 4 .

[7] Ver, por exemplo, cap. 5, sec. 6 da Ref. [8] ou cap. 4 sec. 6.2 da Ref. [2].

[8] S.C. Zilio, Óptica Moderna: Fundamentos e Aplicações, disponível em http://www.fotonica. ifsc.usp.br cap. 3, sec. 5 .

[9] S.L. Gómez, C.S. Lopes, A.A. Bernal-Cardenas, V.M. Lenart e R.F. Turchiello, Rev. Bras. Fis. Tecnol. Apl. 3, 18 (2016).

[10] M.A.C. de Araújo, R. Silva, E. de Lima, D.P. Pereira and P.C. de Oliveira, Appl. Opt. 48, 393 (2009).

[11] M.L. Boas, Mathematical Methods in the Physical Sciences (John Wiley \& Sons, Hoboken, 1983), 2nd ed., cap. 11 , sec. 9 . 